\title{
CAPACIDADE FUNCIONAL DE INDIVÍDUOS APÓS ALTA DA UNIDADE DE TERAPIA INTENSIVA E FATORES ASSOCIADOS
}

\author{
FUNCTIONAL CAPACITY OF INDIVIDUALS AFTER HIGH OF THE UNIT OF \\ INTENSIVE THERAPY AND ASSOCIATED FACTORS
Luciano Magno de Almeida Faria ${ }^{1}$, Rodrigo Santos de Queiroz ${ }^{1}$, Tailani Mendes de Oliveira Araújo $^{2}$, Gustavo Silva Ferreira ${ }^{2}$, Alinne Alves Oliveira ${ }^{1,3}$

1 Fisioterapeuta. Mestre em Ciências da Saúde. Docente da Universidade Estadual do Sudoeste da Bahia - UESB. 2.Fisioterapeuta. graduado pela Universidade Estadual do Sudoeste da Bahia - UESB. 3. Integrante do grupo de pesquisa Integrative Physiology Research da Universidade Estadual do Sudoeste da Bahia (UESB), Jequié, Bahia, Brasil

\begin{abstract}
Objective: To evaluate the functional capacity of individuals using WHODAS 2.0, after 3 years of discharge from the intensive care unit and to identify the associated factors. Methods: Retrospective study with a quantitative approach with cross-sectional design, with a sample of individuals who were discharged three years after of hospitalization in the intensive care unit. Result: Of the individuals in the sample, 64.7\% were women with an average age of 41.6 years. The most frequent primary diagnoses were neurological and respiratory. Of the patients classified by WHODAS 2.0, 47.1\% had moderate to severe disability and $52.9 \%$ had mild or none. Conclusion: Individuals who presented moderate to severe functional disability were associated with longer hospital stays and the use of mechanical ventilation, with the areas of greatest impairment being cognition, life activity and social participation. Individuals classified as having mild or no disability were able to return to work after hospital discharge.
\end{abstract}

Keywords: Artificial respiration; Intensive care; Back to work; Muscle weakness; Daily activities; Mortality

\section{Resumo}

Objetivo: Avaliar a capacidade funcional dos indivíduos utilizando o WHODAS 2.0, após 3 anos de alta da unidade de terapia intensiva $e$ identificar os fatores associados. Métodos: Estudo retrospectivo com abordagem quantitativa com delineamento transversal, tendo como amostra indivíduos que receberam alta hospitalar 3 anos após o internamento na unidade de terapia intensiva. Resultados: Dos indivíduos que compunham a amostra, 64,7\% eram mulheres com idade média 41,6 anos. Os diagnósticos primários mais frequentes foram neurológicos e respiratórios. Dos pacientes classificados pelo WHODAS 2.0, 47,1\% apresentava incapacidade moderada a grave $e$ $52,9 \%$ com deficiência leve ou nenhuma. Conclusão: Indivíduos que apresentaram incapacidade funcional moderada a grave estavam associados ao maior tempo de internação e uso da ventilação mecânica, sendo que as áreas de maiores comprometimentos foram cognição, atividade de vida e participação social. Os indivíduos classificados com incapacidade leve ou nenhuma foram capazes de retornar ao trabalho após a alta hospitalar.

Palavras-chave: Respiração artificial; Cuidados intensivos; Retorno ao trabalho; Debilidade muscular; Atividades cotidianas; Mortalidade. 


\section{Introdução}

Nos últimos anos, milhões de pessoas estão sendo internadas nas Unidades de Terapia Intensiva (UTI), para o tratamento de doenças críticas. ${ }^{(1)}$ Avanços nos cuidados aos pacientes críticos aumentaram a sobrevida dessa população, contudo, esses indivíduos se tornaram suscetíveis a agravos no estado de saúde, interferindo na qualidade de vida e na mortalidade, que podem perdurar até 12 anos da alta hospitalar. ${ }^{(2,3)}$

Os sobreviventes do internamento das UTIs apresentam aumento na incidência de risco de problemas físicos (fraqueza muscular e imobilismo), déficits cognitivos (perdas de memória) e distúrbios mentais (ansiedade e depressão). ${ }^{(4)}$ Estas condições os tornam mais vulneráveis à Síndrome do Cuidado Pós Intensivo (PICS - post intensive care syndrome), que decorre desses agravos, levando-os muitas vezes a reclusão de atividades habituais devido às incapacidades adquiridas durante a internação.

As incapacidades manifestadas após alta das unidades intensivas geram aumento nos custos, pois, muitos desses indivíduos são readmitidos na unidade hospitalar, acarretando em mais despesas para os familiares e/ou governo. ${ }^{(5)}$ As dificuldades na realização das atividades diárias, acarretam no comprometimento do retorno ao trabalho, gerando custos pessoais e sociais relevantes.

Diante desse cenário de condição de saúde, fatores contextuais e de participação social, a Organização Mundial de Saúde (OMS), desenvolveu em 2010, o WHODAS 2.0 (World Health Disability Assessment Schedule), instrumento derivado da Classificação Internacional de Funcionalidade, Incapacidade e Saúde (CIF). Com esta ferramenta, é possível avaliar o nível de funcionalidade em seis domínios de vida: cognição, mobilidade, autocuidado, relações interpessoais, atividades de vida e participação na sociedade. ${ }^{(6,7)}$

Nessa perspectiva, o objetivo deste estudo foi avaliar a capacidade funcional dos indivíduos utilizando o WHODAS 2.0, após 3 anos de alta da unidade de terapia intensiva e identificar os fatores associados.

\section{Metodologia}

Trata-se de um estudo retrospectivo com abordagem quantitativa e possui delineamento transversal.

A população do estudo foi composta por indivíduos que receberam alta hospitalar após histórico de internamento na unidade de terapia intensiva adulto na cidade de Jequié-BA, entre 1 을 de janeiro e 1 o de Maio de 2016, que estavam vivos no período entre 10 de junho a 30 de dezembro de 2018.

Os dados incluídos no estudo foram registrados na primeira etapa da pesquisa, durante a internação dos pacientes. Tais informações foram colhidas do prontuário médico e da fisioterapia. A segunda etapa da pesquisa foi constituída de busca ativa aos pacientes através do contato telefônico, ou do endereço coletados no prontuário. Foi realizada uma visita domiciliar pré-agendada aos indivíduos onde foi aplicado o questionário WHODAS 2.0 e um outro instrumento semiestruturado com perguntas referentes às atividades laborais.

As análises estatísticas foram realizadas com SPSS versão 21.0 para Windows. O teste Shapiro-wilk foi utilizado para testar a normalidade da amostra e em seguida o teste Mann Whitney foi aplicado para comparar as médias das variáveis contínuas. Para os dados categóricos foi utilizado o teste exato de Fisher (quantidade de indivíduos). A significância estatística adotada foi inferior a $5 \%(p<0,05)$ com os resultados relatados como $\mathrm{N}(\%)$ e média (desvio padrão).

Cada um dos 36 itens no WHODAS 2.0 foi pontuado de 0 a 4 . A pontuação total de 144 foi convertida em uma porcentagem como recomenda a OMS. O escore do WHODAS 2.0 foi dicotomizado em nenhum ou incapacidade leve (escore WHODAS de 0 a 24\%) e moderado a incapacidade grave (pontuação no WHODAS de 25 a $100 \%)^{(8,9)}$

O estudo atendeu as determinações da Resolução 466/12 a qual discorre sobre pesquisas envolvendo seres humanos, aceito pelo comitê de ética da Universidade Estadual do Sudoeste da Bahia, sob o número de parecer 2.262814. Os participantes foram explicados e esclarecidos sobre o objetivo da pesquisa e assinaram o termo de consentimento livre e esclarecido (TCLE). 


\section{Resultados}

Dos 93 participantes que compunham a população internada na unidade de terapia intensiva no período da coleta 19,3\% corresponderam os critérios para compor a variável dependente desse estudo (Figura 1).

Figura 1: Composição da amostra de estudo

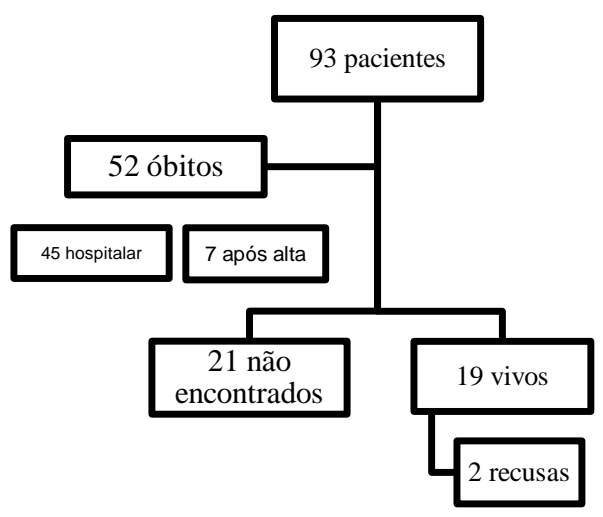

A amostra era composta por $64,7 \%$ mulheres e a média a idade foi de $41,6( \pm 18,6)$ anos. Apenas dois pacientes eram idosos (76 e 89 anos de idade). Os diagnósticos primários mais frequentes foram neurológicos (traumatismo craniano, acidente vascular encefálico) e respiratórios, ambos com $23,5 \%$ dos casos. Dos indivíduos, 70,6\% tinham ocupações como estudos e trabalho antes da doença crítica, enquanto $5,9 \%$ era aposentado e $23,5 \%$ não estavam trabalhando.

A Tabela 1 mostra os dados dos pacientes incluídos no estudo, comparando pacientes dicotomizados em incapacidade moderada a grave e deficiência leve ou nenhuma, 3 anos após o internamento na UTI.
Tabela 1: Análise univariada de dados demográficos de pacientes com incapacidade ligeira ou fraca em comparação com doentes com a incapacidade grave.

\begin{tabular}{cccc}
\hline & Incapaz & Capaz & p \\
Variáveis ordinais & Média & Média & \\
& (DP) & (DP) & \\
\hline Idade & 43,6 & 39,4 & 0,5 \\
Dias de internação & $(18,7)$ & $(19,4)$ & 4 \\
Tempo de ventilação & 14,0 & 8,13 & 0,1 \\
mecânica & $11(10,2)$ & $5,8(7,8)$ & 0,1 \\
Quantidade de & & & 6 \\
comorbidade & $1,7(2,3)$ & $1,6(1,2)$ & 0,6 \\
\hline
\end{tabular}

DP desvio padrão

Nesse estudo pôde observar que o tempo de internação e a maior permanência em ventilação mecânica foram maiores nos pacientes que apresentaram algum nível de incapacidade.

$\mathrm{Na}$ Tabela 2 são mostradas as frequências das variáveis categóricas estudadas segundo o desfecho capacidade definido pelo WHODAS 2.0. Os indivíduos que retornaram ao trabalho, todos foram considerados capazes, isto é, apresentaram nenhuma incapacidade ou incapacidade leve pelo escore e não precisaram ser reinternados. Dos indivíduos considerados incapazes, 55,5\% foram reinternados em hospital no decorrer dos três anos após a alta da UTI. 
Tabela 2: Distribuição dos indivíduos de acordo ao escore do WHODAS 2.0 (totalmente capaz e incapacidade fraca versus incapacidade moderada e grave).

\begin{tabular}{|c|c|c|c|}
\hline \multirow{3}{*}{ Variáveis independentes } & \multicolumn{2}{|c|}{ WHODAS 2.0} & \multirow[b]{3}{*}{$\mathbf{p}$} \\
\hline & Capaz & Incapaz & \\
\hline & n (\%) & n (\%) & \\
\hline Sexo & & & 0,05 \\
\hline Masculino & $5(57,1)$ & $1(11,1)$ & \\
\hline Esteve em VM & & & 0,29 \\
\hline Sim & $5(65,2)$ & $8(88,9)$ & \\
\hline Não & $3(44,8)$ & $1(11,1)$ & \\
\hline Retornou ao trabalho pós alta & & & 0,081 \\
\hline Sim & $4(100)$ & $3(37,5)$ & \\
\hline Não & $0(0)$ & $5(62,5)$ & \\
\hline Reinternação & & & $0,029 *$ \\
\hline Sim & $0(0)$ & $5(55,5)$ & \\
\hline Não & $8(100)$ & $4(44,5)$ & \\
\hline
\end{tabular}

* valor significativo para a análise, VM ventilação mecânica

$\mathrm{Na}$ figura 2, são mostradas as frequências das variáveis dicotomizada (capaz e incapaz) separadas respectivamente pelos seis domínios.
O domínio atividade de vida apresentou 87\% de indivíduos com determinado grau de incapacidade, em contrapartida $37 \%$ relataram incapacidade no domínio relação interpessoal. No quesito mobilidade $50 \%$ da amostra relatou ser capaz.

\section{Discussão}

Figura 2: Frequência dos domínios de acordo a classificação incapacidade e capacidade.

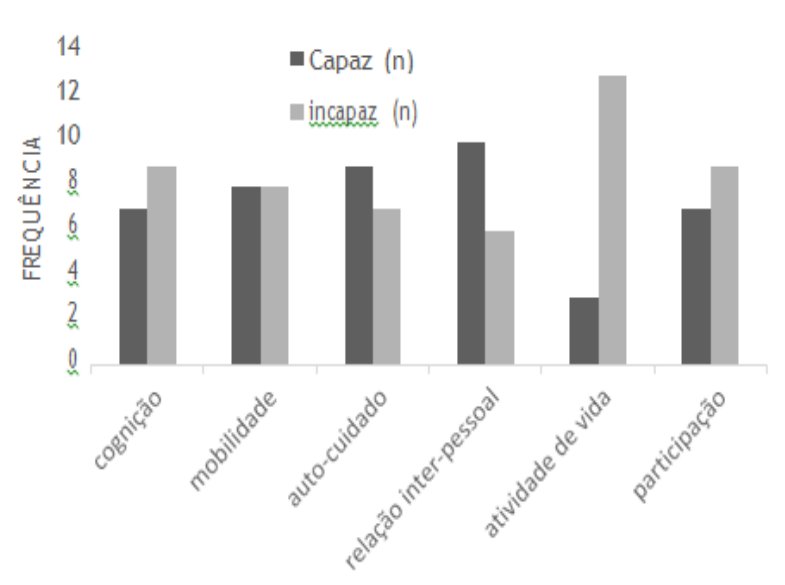

Este estudo analisou a capacidade funcional de indivíduos que estiveram internados na UTI, após um período de três anos da alta, o que o torna original e inovador. Nesse estudo foi possível detectar que os indivíduos com maior tempo de internação e uso prolongado de ventilação mecânica apresentaram algum grau de incapacidade.

Os domínios que apresentaram maior nível de incapacidade foram atividade de vida, cognição e participação social. Além disso, aqueles que retornaram ao trabalho tiveram nenhuma ou incapacidade leve, sendo considerados capazes para suas atividades.

Os indivíduos que apresentaram maior tempo de internação na UTI e em ventilação mecânica prolongada, tiveram algum nível de incapacidade. Esse fato está relacionado às 
alterações da massa óssea e das fibras musculares respiratórias e periféricas geradas pelo desuso, o que dificulta e prolonga a recuperação funcional. Estas alterações também foram encontradas em outros estudos, ${ }^{(10,11)}$ que descreveram o tempo prolongado de internação e uso de ventilação mecânica como preditores de incapacidades funcionais devido à fraqueza adquirida, reduzindo dessa forma, a qualidade e expectativa de vida em médio e longo prazo.

Dos indivíduos considerados incapazes $55,5 \%$, precisaram ser reinternados no período de três anos após a alta da UTI. Esse valor elevado não é encontrado em outros estudos, ${ }^{(12,13)}$ os quais as taxas de reinternações foram inferiores a $20 \%$. Apesar dos fatores serem os mesmos (internação prolongada na unidade e uso de VM), os estudos foram realizados em países desenvolvidos, o que pode interferir nos cuidados, desde o período de internação até o suporte após a alta hospitalar.

A incapacidade influencia na qualidade de vida dos indivíduos restringindo a participação social e o retorno ao trabalho. No nosso estudo, $56,25 \%$ apresentaram incapacidade de participação social e $62,5 \%$ não retornaram as atividades laborais após a alta da UTI, o que pode ser decorrente das alterações cinéticofuncionais. ${ }^{(17)}$ Hodgson et al. ${ }^{(9)}$ em um estudo realizado na Austrália, encontraram resultados próximos ao que encontramos, uma vez que, $60 \%$ da população do estudo não haviam retornado ao trabalho devido à moderada ou grave incapacidade funcional.

Além de alterações musculares, a internação na UTI gera agravos psicológicos que vão da ansiedade à depressão. ${ }^{(18)}$ Observamos que $56,25 \%$ dos indivíduos apresentaram incapacidade cognitiva, achado semelhante ao estudo de Pereira et al. ${ }^{(19)}$, que analisou a ocorrência de comprometimento cognitivo em $48 \%$ das pessoas após 6 meses de alta da UTI. Esse fato pode ser explicado, pois o diagnóstico primário mais prevalente fora de origem neurológica, o que gera déficits cognitivos e físicos, contribuindo para o não retorno ao trabalho mesmo depois de 3 anos de alta.

A incapacidade funcional também é um preditor para mortalidade desde o período de internação até a alta hospitalar, perdurando meses subsequentes. No nosso estudo 51 pacientes que tivemos registro vieram a óbito, reforçando outros achados da literatura, que avaliaram o nível de mortalidade em pacientes que estavam internados e tiveram alta das UTIs, onde sugere que no primeiro ano os índices dos óbitos são elevados em decorrência da condição de saúde. ${ }^{(9,11,14)}$

\section{Considerações finais}

destacar quais as contribuições, implicações, aplicações e recomendações de soluções possíveis para os problemas identificados."

Após três anos da alta hospitalar houve um declínio funcional, sendo que, os indivíduos que tiveram um tempo prolongado de internação e fizeram o uso da ventilação mecânica apresentaram incapacidade moderada à grave. As áreas de maiores comprometimentos foram: cognição, atividade de vida e participação social, sendo que $55,5 \%$ precisaram ser reinternados em hospitais no decorrer desse tempo. Apenas os que tiveram leve ou nenhuma incapacidade, foram capazes de retornar ao trabalho após a alta hospitalar.

Diante do que encontramos, podemos sinalizar a necessidade de manter o indivíduo com seu estado funcional prévio ao evento da internação, ou ganhar a funcionalidade que foi perdida no período da estada hospitalar. Com isso, o indivíduo conseguirá ser reinserido do na sociedade sem demandar muito tempo e custos para a sua recuperação física e cognitiva decorrentes da hospitalização.

As limitações do nosso estudo se deram a partir dos dados coletados nos prontuários, onde nos deparamos com a falta de informações relacionadas aos contatos telefônicos e endereço, dificultado o follow up.

\section{Referências}

1. Society of Critical Care Medicine. Critical care statistics. http://www.sccm. org/Communications/Pages/Critical-

CareStats.aspx. [acesso em abr. 2019]. Disponível em:

https://www.sccm.org/Communications/CriticalCare-Statistics

2. Ohtake PJ, Lee AC, Scott JC, et al. Physical impairments associated with postintensive care syndrome: systematic review based on the World Health Organization's International Classification of Functioning, Disability and Health Framework. Phys Ther. 2018; 98:631-45.

3. Altman $M T$, Knauert MP, Murphy $T E$, Ahasic AM, Chauhan Z, Pisani MA, Association of intensive care unit delirium with sleep disturbance and functional disability after critical 
illness: an observational cohort study. Ann Intensive Care. 2018; 8; 1:8.

4. Gayat E, Cariou A, Deye N, VieillardBaron A, Jaber S, Damoisel C, et al. Determinants of long-term outcome in ICU survivors: results from the FROG-ICU study. Crit Care 2018; 22:8.

5. Hermans $G$, Van Mechelen H, Clerckx B, Vanhullebusch T, Mesotten D, Wilmer A, et al. Acute outcomes and 1-year mortality of ICUacquired weakness: a cohort study and propensity matched analysis. Am J Respir Crit Care Med. 2014;190: 410-20.

6. Ferrer MLP, Perracini MR, Rebustini $F$, Buchalla CM. WHODAS 2.0-BO: dados normativos para avaliação de incapacidade em idosos. Rev Saúde Pública. 2019; 53;19.

7. World Health Organization. Measuring Health and Disability: Manual for WHO Disability Assessment Schedule (WHODAS 2.0). Geneve: WHO, 2010.

8. Shulman MA, Myles PS, Chan MT, Mcllroy DR, Wallace S, Ponsford J. Measurement of disability-free survival after surgery. Anesthesiology 2015;122; 524:536.

9. Hodgson $C L$, Udy AA, Bailey $M$, Barrett J, Bellomo R, Bucknall $T$, et al. The impact of disability in survivors of critical illness. Intensive Care Med. 2017; 43; 992:1001

10. Hodgson CL, Tipping CJ. Physiotherapy management of intensive care unit-acquired weakness. J Physiother. 2017; 63; 4:10.

11. Wilson ME, Barwise A, Heise $\mathrm{KJ}$, Loftsgard TO, Dziadzko M, Cheville A, et al. Longterm return to functional baseline after mechanical ventilation in the ICU. Crit Care Med. 2018; 46; 562:9

12. Liu CL, Chen WJ, Su J, Yang YH, Lin CY, Lin RL. Characteristics and outcomes of patients readmitted to the medical intensive care unit: a retrospective study in a tertiary hospital in Taiwan. Int J Gerontol. 2017; 11; 244:248

13. Nguyen MC, Strosberg DS, Jones TS, et al. Mortality and readmission of outcomes after discharge from the surgical intensive care unit to long-term, acute-care hospitals. Surgery. 2017;161;1367:1375

14. Ohtake PJ, Coffey Scott J, Hinman RS, Lee AC, Smith JM. Impairments, activity limitations and participation restrictions experienced in the first year following a critical illness: protocol for a systematic review. BMJ Open. 2017; 7:e013847.

15. Rydingsward JE, Horkan CM, Mogensen KM, Quraishi AS, Amrein K, Christopher KB. Functional status in ICU survivors and out of hospital outcomes: a cohort study. Crit Care Med. 2016; 44; 869:879
16. Hashem MD, Nallagangula A, Nalamalapu S, Nunna K, Nausran U, Robinson KA, et al. Patient outcomes after critical illness: a systematic review of qualitative studies following hospital discharge. Crit Care. 2016; 20; 345.

17. Pernambuco AP, Lana RDC, Ribeiro DDC, Polese JC. Utilização da Classificação Internacional de Funcionalidade, Incapacidade e Saúde na terapia intensiva. In: Martins JA, Dias CM, Andrade FMD. PROFISIO - Programa de atualização em fisioterapia em terapia intensiva adulto: Ciclo 5. Porto Alegre: Artmed. 2015; 11134.

18. Choi J, Tate JA, Rogers MA, Donahoe MP, Hoffman LA. Depressive symptoms and anxiety in intensive care unit (ICU) survivors after ICU discharge. Heart Lung. 2016; 45;140:146.

19. Pereira $\mathrm{S}$ et al. Desfechos psicológicos em longo prazo após alta da terapia intensiva. Rev Bras Ter Intensiv. 2018; 30; 28:34

\section{Endereço para Correspondência}

\section{Alinne Alves Oliveira}

Endereço: Av. José Moreira Sobrinho, S/N. Jequiezinho - 45.200-000 - Jequié - BA, Brasil. E-mail: alinnealvesoliveira@uesb.edu.br

Recebido em 19/01/2021

Aprovado em 15/05/2021

Publicado em 15/06/2021 